\title{
Articular Muscle
}

National Cancer Institute

\section{Source}

National Cancer Institute. Articular Muscle. NCI Thesaurus. Code C32145.

A muscle that inserts directly onto the capsule of a joint, acting to retract the capsule in certain movements. 